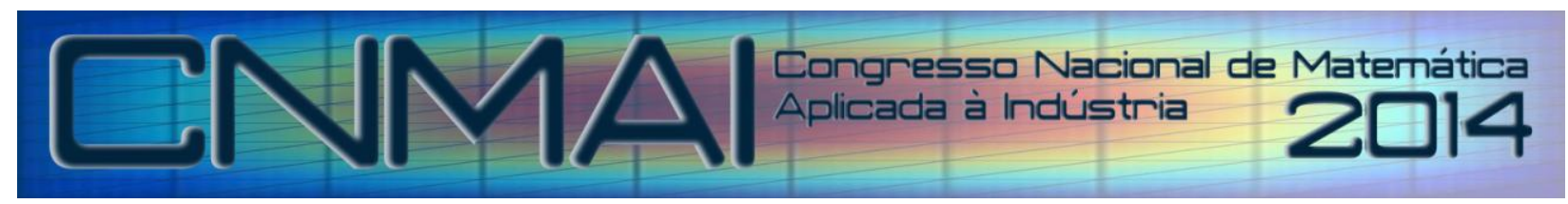

18 a 21 de novembro de 2014, Caldas Novas - Goiás

\title{
MODELO DE OTIMIZAÇÃO LINEAR PARA DEFINIÇÃO DA EQUIPE DE TRABALHO DE ABRIGOS MUNICIPAIS DA ASSISTÊNCIA SOCIAL DE CAMPINAS
}

\author{
Paulo Eduardo Bassi Arce, paulob.eduardo@gmail.com ${ }^{1}$ \\ ${ }^{1}$ Universidade de São Paulo - Escola de Engenharia de São Carlos, Departamento de Engenharia Elétrica e \\ Computação - Av. Trabalhador São-carlense, 400
}

\begin{abstract}
Resumo: O município de Campinas conta com uma vasta rede de equipamentos de Assistência Social, os quais ofertam diversos serviços. Entre estes, está o abrigo institucional, o qual abriga crianças e adolescentes em situação de vulnerabilidade social. Os abrigos institucionais contam com grande estrutura logística e de recursos humanos, os quais devem ser adequadamente dimensionados, visando eficiência econômica e uso racional dos recursos públicos. Os requisitos necessários à composição das equipes de trabalho dos abrigos encontram-se definidos na Norma Operacional Básica do Sistema Único de Assistência Social (NOB-RH SUAS). No entanto, seu dimensionamento e a alocação de funcionários são tarefas críticas e não descritas na NOB. Dessa forma, faz-se necessária uma ferramenta de suporte à decisão, que auxilie o gestor local na composição e alocação da equipe de trabalho. Assim, este trabalho propõe um modelo de otimização linear, tendo como objetivo a minimização da função número de funcionários com uma restrição do número de profissionais mínimo e levando em conta as diversas especialidades exigidas. Ambas as restrições são obtidas na NOB. Esta metodologia de tratamento do problema corresponde a uma abordagem pioneira dentro da Assistência Social, uma vez que não há registros na literatura científica de trabalhos de otimização e pesquisa operacional no dimensionamento de equipamentos da Assistência Social. O modelo é implementado computacionalmente via o solver LINGO 7. Para facilitar o uso da ferramenta pelo gestor local, o programa utiliza o MS Excel para fazer interface com o LINGO 7, já que o Excel é uma ferramenta amplamente difundida e familiar aos usuários locais. Os resultados práticos do trabalho devem levar a uma melhor gestão de RH nos equipamentos em que venham a ser implementados.
\end{abstract}

Palavras-chave:Otimização Linear, Gestão de Recursos Humanos, Gestão Pública, Assistência Social

\section{INTRODUÇÃ̃O}

No Brasil, a Assistência Social é definida e regulamentada pela lei orgânica de assistência social (MDS, 1993), e instrumentalizada por meio do Sistema Único de Assistência Social (SUAS) (MDS, 2006). Entre as diversas diretrizes que regulamentam o SUAS, encontra-se a Norma Operacional Básica de Recursos Humanos do SUAS (NOB-RH SUAS). Nesta normativa encontram-se definidos e tipificados os serviços prestados pela assistência social, executados regionalmente pelos municípios. Assim, cada serviço apresenta especificidades metodológicas que visam atender a um público determinado em um contexto de exposição a vulnerabilidades ou riscos sociais.

Dentre as diversas especificidades metodológicas que caracterizam os serviços de assistência social, encontram-se os quadros funcionais de recursos humanos necessários para executar determinado serviço social. Os quadros funcionais descritos na NOB-RH SUAS são elaborados levando em consideração dois aspectos principais: i) a especialidade, em termos de formação, do profissional desejado para determinado cargo; e ii) a quantidade de usuários a ser atendida por determinado serviço. Com estes dois aspectos a NOB-RH SUAS define qual a relação entre usuários atendidos e profissionais necessários, para cada cargo e para cada serviço.

Neste trabalho será apresentado um modelo de otimização linear que visa minimizar o número de funcionários necessários em um abrigo institucional em função do número de usuários. No entanto, o presente estudo pode ser reproduzido para qualquer outro serviço da assistência social que esteja tipificado na NOB-RH SUAS. A escolha do abrigo institucional se deve a sua importância como serviço social, à sua elevada complexidade e ao elevado número de profissionais necessários. No município de Campinas, embora haja uma vasta rede de parceiros privados no serviço de abrigo institucional, o setor público conta com apenas um abrigo, o Centro Municipal de Proteção à Criança e ao Adolescente (CMPCA). Os resultados apresentam a quantidade ótima de funcionários em função ao número de metas 
necessário à adequação do CMPCA à NOB-RH SUAS. Estes resultados podem ser utilizados para compor o planejamento estratégico da gestão deste tipo de serviço, que é de vital importância para a garantia do direito à proteção social para todos os cidadãos e que encontra no CMPCA sua única representação pública dentro do município de Campinas. A abordagem proposta é inovadora dentro da Assistência Social, sendo este um trabalho original dentro da área. Desta forma, não serão encontrados trabalhos que utilizem técnicas de pesquisa operacional aplicada ao dimensionamento proposto. Espera-se que este trabalho constitua parte do "estado da arte" neste tema.

\section{METODOLOGIA PROPOSTA}

\subsection{Programação Linear}

A resolução matemática do problema será dada através de uma formulação de um modelo de otimização linear. Uma breve descrição deste assunto será feita a seguir.

Basicamente, um problema de otimização linear ou programação linear (PL), é um problema de otimização onde a função objetivo e as restrições são lineares (Lachtermacher, 2005). Matematicamente, tem-se:

$$
\begin{aligned}
& \text { maximizar } z=\sum_{j=1}^{p} c_{j} x_{j} \\
& \text { sujeito } a \\
& \sum_{j=1}^{p} a_{i j} x_{j} \leq b_{i}, i=1,2, \ldots, q \\
& x_{j} \geq 0, j=1,2, \ldots, p
\end{aligned}
$$

Nas equações de (1) a (3) $c_{j}, a_{i j}$ e $b_{i}$ são dados (números reais) e $x_{j}$ representa as variáveis de decisão. A função linear a ser maximizada em (1) é denominada função objetivo. As restrições de não negatividade são conhecidas como triviais.

Alternativamente é possível modelar o problema apresentado acima como um problema de minimização, onde a função objetivo será minimizada, que é o caso deste trabalho.

\subsection{Determinação dos Parâmetros}

Nesta seção serão determinados os parâmetros necessários para realizar a modelagem do problema de acordo com as equações (1) a (3).

Conforme citado, a NOB-RH SUAS define o número mínimo de profissionais necessários para cada serviço em função do número de usuários a serem atendidos. Neste sentido a NOB-RH/SUAS define as Equipes de Referência como "equipes constituídas por servidores efetivos responsáveis pela organização e oferta de serviços, programas, projetos e benefícios de proteção social básica e especial, levando-se em consideração o número de famílias $e$ indivíduos referenciados, o tipo de atendimento e as aquisições que devem ser garantidas aos usuários (MDS, 2006)"

A Tabela 1 define o quadro funcional para o serviço do tipo abrigo institucional:

Tabela 1. Quadro funcional para serviço de Abrigo Institucional. Fonte: (MDS, 2006)

\begin{tabular}{|l|l|l|}
\hline Profissional/Função & Escolaridade & Quantidade \\
\hline Coordenador & Nível superior ou médio & $\begin{array}{l}1 \text { profissional referenciado para até 20 } \\
\text { usuários acolhidos em, no máximo, 2 } \\
\text { equipamentos. }\end{array}$ \\
\hline Cuidador & $\begin{array}{l}\text { Nível médio e qualificação } \\
\text { específica } \\
\text { profissional para até 10 usuários, por } \\
\text { turno. A quantidade de cuidador por } \\
\text { usuário deverá ser aumentada quando } \\
\text { houver usuários que demandem atenção } \\
\text { específica (com deficiência, com } \\
\text { necessidades específicas de saúde, } \\
\text { pessoas soropositivas, idade inferior a } \\
\text { um ano, pessoa idosa com Grau de } \\
\text { Dependência II ou III, dentre outros). } \\
\text { Para tanto, deverá ser adotada a seguinte } \\
\text { relação: }\end{array}$ \\
\hline
\end{tabular}




\begin{tabular}{|c|c|c|}
\hline & & $\begin{array}{l}\text { a) } 1 \text { cuidador para cada } 8 \text { usuários, } \\
\text { quando houver } 1 \text { usuário com demandas } \\
\text { específicas; } \\
\text { b) } 1 \text { cuidador para cada } 6 \text { usuários, } \\
\text { quando houver } 2 \text { ou mais usuários com } \\
\text { demandas específicas. }\end{array}$ \\
\hline Auxiliar Cuidador & $\begin{array}{l}\text { Nível fundamental e qualificação } \\
\text { específica }\end{array}$ & $\begin{array}{l}1 \text { profissional para até } 10 \text { usuários, por } \\
\text { turno. A quantidade de cuidador usuário } \\
\text { deverá ser aumentada quando houver } \\
\text { usuários que demandem atenção } \\
\text { específica (com deficiência, com } \\
\text { necessidades específicas de saúde, } \\
\text { pessoas soropositivas, idade inferior a } \\
\text { um ano, pessoa idosa com Grau de } \\
\text { Dependência II ou III, dentre outros). } \\
\text { Para tanto, deverá ser adotada a seguinte } \\
\text { relação: } \\
\text { a) } 1 \text { auxiliar de cuidador para cada } 8 \\
\text { usuários, quando houver } 1 \text { usuário com } \\
\text { demandas específicas; } \\
\text { b) } 1 \text { auxiliar de cuidador para cada } 6 \\
\text { usuários, quando houver } 2 \text { ou mais } \\
\text { usuários com demandas específicas. }\end{array}$ \\
\hline Assistente Social & Nível Superior & $\begin{array}{l}1 \text { profissional para atendimento a, no } \\
\text { máximo, } 20 \text { usuários acolhidos em até } \\
\text { dois equipamentos da alta complexidade } \\
\text { para pequenos grupos. }\end{array}$ \\
\hline Psicólogo & Nível Superior & $\begin{array}{l}1 \text { profissional para atendimento a, no } \\
\text { máximo, } 20 \text { usuários acolhidos em até } \\
\text { dois equipamentos da alta complexidade } \\
\text { para pequenos grupos. }\end{array}$ \\
\hline
\end{tabular}

Tendo em vista o estudo de caso abordado (abrigo municipal CMPCA), o dimensionamento de funcionários será feito para os cargos de Cuidador e Auxiliar Cuidador, pois para os demais cargos a demanda é menor, e não há necessidade de trabalho em turnos. Os dois cargos alvos do presente estudo apresentam maior demanda e também maior problemática já que, embora apresentem requisitos diferentes, são executados pelo mesmo profissional (monitor).

Assim, deste ponto em diante, será considerado o auxiliar de cuidador como um cuidador também. Outra consideração a ser feita diz respeito à relação profissional/usuário. Conforme a tabela 1 são três relações possíveis. Neste trabalho será adotada a relação intermediária, de 1 Cuidador para 8 usuários e 1 Auxiliar Cuidador para 8 usuários. Portanto tem-se 2 Cuidadores para 8 usuários. Logo, define-se a relação profissional/usuário na equação (4):

$$
r=\frac{\text { profissional }}{\text { usuários }}=\frac{2}{8}=\frac{1}{4}
$$

O número de usuários é um termo variável, podendo ser interpretado como um parâmetro no processo de otimização que irá influenciar no número de funcionários necessários. Além disso, deve-se considerar que cada funcionário trabalha cinco dias da semana e descansa dois, em jornadas diárias de oito horas. Portanto para atender às 24 horas de funcionamento diário do CMPCA, serão necessários três turnos de trabalho (manhã, tarde e noite).

\subsection{Modelo Proposto}

Para a resolução do problema proposto o mesmo será modelado em relação ao dia de início da jornada semanal. Assim, podem-se ajustar as variáveis de decisão como o número de empregados que iniciam a jornada semanal em cada dia da semana:

$$
\begin{aligned}
& N_{1} \text { - Iniciaram as atividades no domingo } \\
& N_{2} \text { - Iniciaram as atividades na segunda } \\
& N_{3} \text { - Iniciaram as atividades na terça } \\
& N_{4}-\text { Iniciaram as atividades na quarta } \\
& N_{5}-\text { Iniciaram as atividades na quinta } \\
& N_{6}-\text { Iniciaram as atividades na sexta } \\
& N_{7}-\text { Iniciaram as atividades no sábado }
\end{aligned}
$$


Portanto, o número de funcionários que trabalhaem um dia seria a soma do número de empregados que começariam a trabalhar 4, 3, 2, 1 dia antes e no próprio dia. Matemáticamente, isso se traduz em:

$$
\begin{aligned}
& N_{1}+N_{2}+N_{3}+N_{4}+N_{5}-\text { Trabalham na quinta }- \text { feira } \\
& N_{2}+N_{3}+N_{4}+N_{5}+N_{6}-\text { Trabalham na sexta }- \text { feira } \\
& N_{3}+N_{4}+N_{5}+N_{6}+N_{7}-\text { Trabalham no sábado } \\
& N_{4}+N_{5}+N_{6}+N_{7}+N_{1}-\text { Trabalham no domingo } \\
& N_{5}+N_{6}+N_{7}+N_{1}+N_{2}-\text { Trabalham na segunda }- \text { feira } \\
& N_{6}+N_{7}+N_{1}+N_{2}+N_{3}-\text { Trabalham na terça }- \text { feira } \\
& N_{7}+N_{1}+N_{2}+N_{3}+N_{4}-\text { Trabalham na quarta }- \text { feira }
\end{aligned}
$$

Conforme citado anteriormente, o objetivo do trabalho é minimizar o número de funcionários do abrigo. Assim, a função objetivo é definidaem (5):

$$
\min N_{1}+N_{2}+N_{3}+N_{4}+N_{5}+N_{6}+N_{7}
$$

As únicas restrições do problema dizem respeito ao número mínimo de funcionários por dia. O número mínimo de funcionários por dia é obtido pela relação apresentada na equação (4) e pelo número de usuários, de modo que as restrições se tornam:

$$
\begin{aligned}
& N_{1}+N_{2}+N_{3}+N_{4}+N_{5} \geq p \\
& N_{2}+N_{3}+N_{4}+N_{5}+N_{6} \geq p \\
& N_{3}+N_{4}+N_{5}+N_{6}+N_{7} \geq p \\
& N_{4}+N_{5}+N_{6}+N_{7}+N_{1} \geq p \\
& N_{5}+N_{6}+N_{7}+N_{1}+N_{2} \geq p
\end{aligned}
$$

Onde $p$ corresponde a um parâmetro que relaciona a relação $r$ da equação (4) com o número de usuários $n$ de acordo com a equação (11) :

$$
p=r * n
$$

A Figura 1 resume o processo realizado neste trabalho:

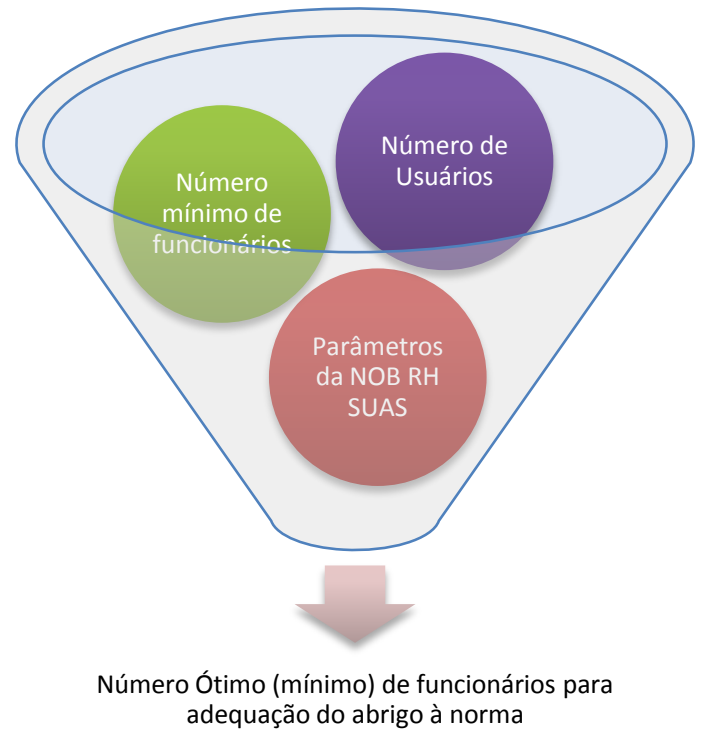

Figura 1. Resumo do processo de otimização.

\section{RESULTADOS}

Nesta seção serão apresentados os resultados obtidos pela modelagem proposta. Para a resolução do problema, foi utilizado o software LINGO 7, que é um solver linear e não-linear, em conjunto com o MS Excel para manipulação dos dados. Ressalta-se que nesta etapa, foi realizada a otimização de um problema contínuo.

Os resultados foram obtidos realizando diversas rodadas do modelo de otimização, variando o parâmetro $p$ através do número de usuários $n$.

A Figura 2 apresenta o número ótimo de funcionários em função ao número de usuários. Os valores ótimos de número de funcionários apresentados na Figura 2 correspondem à quantidade de funcionários necessários para a 
composição da equipe de trabalho do abrigo, de modo que haja o número mínimo de funcionários para cada dia da semana.

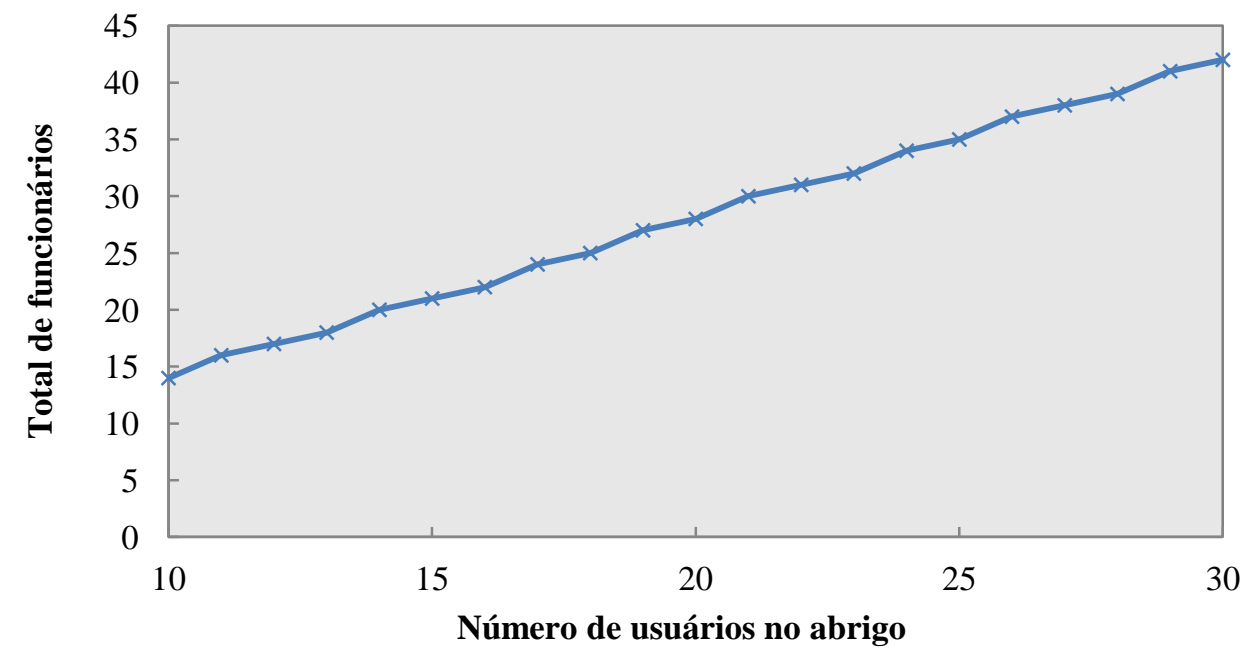

Figura 2. Número de funcionários na equipe de trabalho do abrigo municipal em função do número de usuários.

Cada ponto da Figura 2 representa um par ótimo de funcionários x usuários. Assim, são todas combinações ótimas. As rodadas foram realizadas variando o número de usuários em uma faixa de valores entre 10 e 30 usuários. Esta faixa de valores foi escolhida por conter o número mínimo de usuários (10) e por apresentar o número médio de usuários do abrigo (30).

Por se tratar de um problema de otimização linear, espera-se que a Figura 2 seja uma reta. No entanto neste caso, uma reta incluiria valores decimais, os quais não são compatíveis com o variável número de funcionários. Assim, os desvios em relação à reta ótima visam aproximar o valor ótimo obtido de um número inteiro, compatível com a variável número de funcionários. Embora o modelo encontre os valores ótimos, para adaptar os valores obtidos à característica inteira da variável número de funcionários, a Figura 2 apresenta resultados aproximados, que são sub-ótimos.

\section{CONCLUSÃ̃o}

\subsection{Considerações Finais}

O presente trabalho tratou do problema de dimensionamento de equipe de trabalho de abrigos institucionais de crianças e adolescentes na assistência social. Para resolver o problema utilizou-se um modelo de otimização linear, obtendo o número ótimo de funcionários para determinado número de usuários. Esta abordagem ao problema é um trabalho pioneiro na área de Assistência Social. O resultado pode ser utilizado como uma justificativa técnica para a contratação de novos funcionários de modo a adequar os abrigos à norma da NOB-RH SUAS. Neste trabalho utilizouse o CMPCA como estudo de caso, mas o trabalho se estende a qualquer outro abrigo da rede socioassistencial.

$\mathrm{O}$ correto dimensionamento do número de funcionários no abrigo deve levar a ganhos em termos de qualidade de prestação do serviço, melhorando a assistência ao público exposto à vulnerabilidade social. Além disso, o correto dimensionamento do quadro funcional deve levar a significativos ganhos financeiros uma vez que se espera desta maneira reduzir a necessidade de horas extra.

\subsection{Continuidade do trabalho}

Além de dimensionar adequadamente o número de funcionários necessários para a adequação do abrigo municipal aos critérios estabelecidos pela NOB-RH, é possível propor um sistema automático de elaboração da escala de trabalho. Em outras palavras, propõe-se a criação de um sistema que gere automaticamente as escalas de trabalho do abrigo, distribuindo nos três turnos (manhã, tarde e noite) o número ótimo de funcionários obtido na primeira etapa. Este procedimento evitaria que o gestor local realize a elaboração da escala de trabalho de forma manual.

A elaboração da escala de trabalho é um fator crítico dentro de atendimentos 24 horas. Diversos autores abordam a questão da elaboração automática de escalas de trabalho, como pode ser apreciado em (Rangel, 2007), (Anzai, 1987), (Irvin, 1999) e diversos outros. A vasta literatura acadêmica e científica a respeito do assunto denota sua importância dentro das organizações. Em comum, estes trabalhos apresentam o fato de lidarem com esta questão através de modelagens de programação linear inteira, com restrições referentes às legislações trabalhistas vigentes e parâmetros de preferências de folga na escala. 
A Figura 3 apresenta a proposta de continuidade do trabalho. A primeira parte, que corresponde ao trabalho desenvolvido neste artigo, encontra-se referenciada como Primeira Etapa. A Segunda Etapa corresponde à continuidade do trabalho, a qual está atualmente em desenvolvimento.

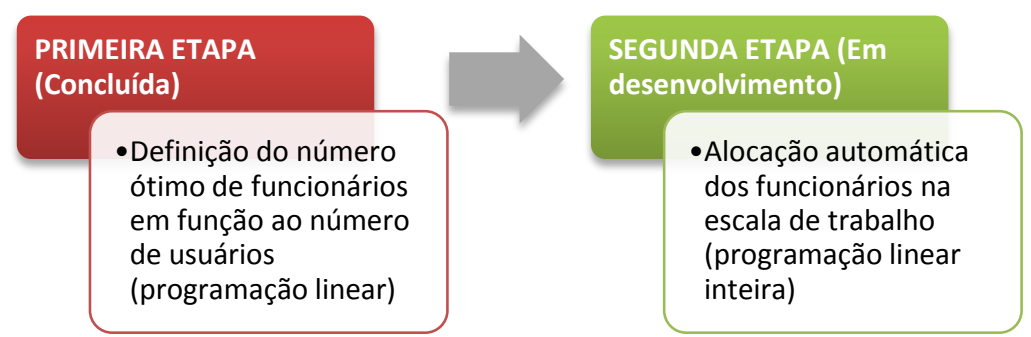

Figura 3. Proposta de continuidade do trabalho.

\subsubsection{Resultados Preliminares da Segunda Etapa}

A modelagem matemática dada à segunda parte é apresentada a seguir. Em linhas gerais o objetivo é alocar os funcionários ao longo dos 30 ou 31 dias do mês de modo que todos trabalhem o número de dias úteis correspondente ao mês em questão, e tenham as folgas referentes a finais de semana e feriados. Além disso, deve-se na medida do possível atender aos pedidos de folga dos funcionários. Assim, propõe-se a seguinte modelagem:

$$
\begin{aligned}
& \min \sum_{i} \sum_{j} X_{i j} F_{i j} \\
& \text { sujeito } a: \\
& \sum_{j} X_{i j}=d_{u} \\
& \sum_{i} X_{i j}=n_{\min } \\
& X_{i j}=\begin{array}{r}
1, \text { se o funcionário } i \text { for trabalhar no dia } j \\
0, \text { se o funcionário } i \text { for folgar no dia } j
\end{array}
\end{aligned}
$$

Onde $X_{i j}$ corresponde à variável de decisão que atribui 1 para trabalho e 0 para folga, $F_{i j}$ corresponde à intenção de folga do funcionário $i$ no dia do mês $j$. Assim a função objetivo é minimizar o produto entre a variável de trabalho e a intenção de folga. Desta maneira a otimização privilegia as folgas (que têm peso maior) atribuindo o valor 0 no dia correspondente aos dias de maior peso (intenção de folga). As restrições (13) e (14) dizem respeito ao número correto de dias trabalhados no mês referentes aos dias úteis $\left(d_{u}\right)$ e ao número mínimo de funcionários por dia para o abrigo $\left(n_{\min }\right)$ respectivamente. O $n_{\min }$ é obtido na Primeira Etapa, conforme referenciado na Figura 3 . A Tabela 2 ilustra os resultados preliminares:

Tabela 2. Comparação entre a intenção de folga e a escala criada.

\begin{tabular}{|l|r|r|r|r|r|r|r|r|r|r|r|r|r|r|r|}
\hline $\begin{array}{l}\text { INTENÇÃO } \\
\text { DE FOLGA }\end{array}$ & dom & seg & ter & qua & qui & sex & sab & dom & seg & ter & qua & qui & sex & sáb & dom \\
\hline Fij & $\mathbf{1}$ & $\mathbf{2}$ & $\mathbf{3}$ & $\mathbf{4}$ & $\mathbf{5}$ & $\mathbf{6}$ & $\mathbf{7}$ & $\mathbf{8}$ & $\mathbf{9}$ & $\mathbf{1 0}$ & $\mathbf{1 1}$ & $\mathbf{1 2}$ & $\mathbf{1 3}$ & $\mathbf{1 4}$ & $\mathbf{1 5}$ \\
\hline $\begin{array}{l}\text { funcionário } \\
\mathbf{1}\end{array}$ & 25 & 1 & 1 & 1 & 1 & 1 & 50 & 25 & 1 & 1 & 1 & 1 & 100 & 1 & 1 \\
\hline $\begin{array}{l}\text { funcionário } \\
\mathbf{2}\end{array}$ & 1 & 1 & 100 & 100 & 100 & 1 & 1 & 25 & 1 & 100 & 1 & 1 & 1 & 50 & 25 \\
\hline $\begin{array}{l}\text { funcionário } \\
\mathbf{3}\end{array}$ & 25 & 1 & 1 & 1 & 1 & 100 & 50 & 1 & 100 & 100 & 1 & 100 & 100 & 1 & 1 \\
\hline $\begin{array}{l}\text { funcionário } \\
\mathbf{4}\end{array}$ & 1 & 1 & 1 & 1 & 1 & 1 & 50 & 25 & 1 & 1 & 100 & 1 & 1 & 1 & 1 \\
\hline $\begin{array}{l}\text { funcionário } \\
\mathbf{5}\end{array}$ & 1 & 1 & 1 & 1 & 1 & 1 & 1 & 1 & 1 & 1 & 1 & 1 & 1 & 1 & 1 \\
\hline & & & & & & & & & & & & & & & \\
\hline
\end{tabular}




\begin{tabular}{|l|r|r|r|r|r|r|r|r|r|r|r|r|r|r|r|}
\hline & & & & & & & & & & & & & & & \\
\hline ESCALA & dom & seg & ter & qua & qui & sex & sab & dom & seg & ter & qua & qui & sex & sáb & dom \\
\hline Xij & $\mathbf{1}$ & $\mathbf{2}$ & $\mathbf{3}$ & $\mathbf{4}$ & $\mathbf{5}$ & $\mathbf{6}$ & $\mathbf{7}$ & $\mathbf{8}$ & $\mathbf{9}$ & $\mathbf{1 0}$ & $\mathbf{1 1}$ & $\mathbf{1 2}$ & $\mathbf{1 3}$ & $\mathbf{1 4}$ & $\mathbf{1 5}$ \\
\hline $\begin{array}{l}\text { funcionário } \\
\mathbf{1}\end{array}$ & 0 & 1 & 1 & 1 & 1 & 1 & 0 & 0 & 1 & 1 & 1 & 1 & 0 & 1 & 1 \\
\hline $\begin{array}{l}\text { funcionário } \\
\mathbf{2}\end{array}$ & 1 & 1 & 0 & 0 & 0 & 1 & 1 & 0 & 1 & 0 & 1 & 1 & 1 & 0 & 0 \\
\hline $\begin{array}{l}\text { funcionário } \\
\mathbf{3}\end{array}$ & 0 & 1 & 1 & 1 & 1 & 0 & 0 & 1 & 0 & 0 & 1 & 0 & 0 & 1 & 1 \\
\hline $\begin{array}{l}\text { funcionário } \\
\mathbf{4}\end{array}$ & 1 & 1 & 1 & 1 & 1 & 1 & 1 & 1 & 1 & 1 & 0 & 1 & 1 & 1 & 1 \\
\hline $\begin{array}{l}\text { funcionário } \\
\mathbf{5}\end{array}$ & 1 & 1 & 1 & 1 & 1 & 1 & 1 & 1 & 1 & 1 & 1 & 1 & 1 & 1 & 1 \\
\hline
\end{tabular}

Conforme indica a Tabela 2, a maioria dos pedidos de folga é atendida. Na tabela de intenção de folga (Fij) atribuem-se diferentes pesos para os dias, visando estabelecer um critério de preferência dos dias de folga. Os próximos passos na Segunda Etapa são a implementação do código no LINGO 7, e a criação de uma interface amigável ao usuário. Também será realizada a implementação de outras restrições referentes às legislações trabalhistas e o aumento de número de variáveis, já que os abrigos contam com muito mais do que 5 funcionários. O resultado aqui apresentado é apenas um indicativo do trabalho futuro.

\section{REFERÊNCIAS}

ANZAI, M., MIURA, Y. Computer program for quick work scheduling of nursing staff. Med Inform. 12(1): 43-52 1987.

IRVIN A.S., BROWN H.N. Self-Scheduling with Microsoft Excel. Nurs Econ;17(4):201- 206, 1999.

LACHTERMACHER, G. Pesquisa Operacional na Tomada de Decisões. São Paulo: Elsevier, 2005.

MINISTÉRIO DO DESENVOLVIMENTO SOCIAL (MDS). 1993. LOAS Lei Orgânica da Assistência Social. http://www.mds.gov.br/assistenciasocial/secretaria-nacional-de-assistencia-social-snas/livros/loas-lei-organica-deassistencia-social/loas-lei-organica-de-assistencia-social

MINISTÉRIO DO DESENVOLVIMENTO SOCIAL (MDS). 2006. NOB-RH SUAS Norma Operacional Básica de Recursos Humanos do Sistema Único de Assistência Social. http://www.mds.gov.br/assistenciasocial/publicacoespara-impressao-em-grafica/norma-operacional-basica-de-recursos-humanos-do-suas-nob-rh-suas

RANGEL, A. L., ÉVORA, Y. D. M. Elaboração automática da escala periódica de trabalho dos profissionais de enfermagem por meio de um software específico. Revista eletrônica de enfermagem, v09, n 01, p. 17-30, 2007.

\section{RESPONSABILIDADE AUTORAL}

“O(s) autor(es) é(são) o(s) único(s) responsável(is) pelo conteúdo deste trabalho”. 


\section{LINEAR OPTIMIZATION MODEL FOR DEFINITION OF WORKING STAFF IN SOCIAL ASSISTANCE SHELTERS}

\section{CNMAI2014-0012}

Abstract. The municipality of Campinas has a vast network of social assistance equipment, which proffer diverse services. Among these is the institutional shelter, which houses children and adolescents in situations of social vulnerability. Institutional shelters come with extensive logistics infrastructure, human resources, which must be appropriately sized, seeking economic efficiency and rational use of public resources. The requirements for the composition of work teams shelters are defined in the Basic Operational Unified Social Assistance (NOB-RH SUAS). However, their dimensioning and allocation of staff is a critical task and not described in NOB. Thus, it is necessary a tool for decisions support that helps the local manager in the composition and allocation of the work team. Thus, this paper proposes a linear optimization model, with the objective of minimizing the number of employees due to a restriction on the minimum number of professionals and taking into account the various specialties required. Both restrictions are obtained in NOB. The model computationally via the solver LINGO 7 is implemented. To facilitate use of the tool by the local manager, the program uses MS Excel to interface with LINGO 7, since Excel is a widespread and familiar to local users tool. The practical results of the work should lead to better management of HR in equipment that might be implemented.

Keywords: Linear Optimization, Human Resource Management, Public Administration, Social Assistance 